\title{
Un centro di eccellenza nefrologica italiano: l'esperienza di una specializzanda
}

\author{
Stefania Aresu
}

Dipartimento di Nefrologia, Dialisi e Trapianto di Rene, Ospedale San Michele - Università degli Studi di Cagliari, Cagliari

\begin{abstract}
A young Nephrologist's experience in an Italian Center of EXCEllence for Nephrology
Abstract. Participating to an international congress is always an interesting and exciting experience for young residents. In particular, in my case, the congress of Vicenza was an opportunity to plan a fellowship beyond the classical training program and to start an experience in the field of research at the Nephrology/IRRIV of San Bortolo Hospital in Vicenza. I was able to experience a multicultural and interdisciplinar reality that fascinated and inspired me for its practical applications in various research fields and in the designing of dialysis machines.
\end{abstract}

Key words: Acute kidney injury, Scientific research, Multidisciplinarity

Conflict of interest: None.

Financial support: None.

Accettato: 9 Luglio 2014

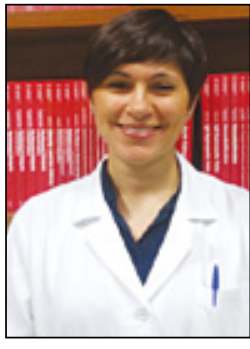

Stefania Aresu

\section{Introduzione}

Il percorso formativo di noi specializzandi della scuola di Specializzazione di Nefrologia presso l'Università di Cagliari non si limita alla frequenza in reparto ma è spesso coadiuvato dalla partecipazione a corsi di formazione e congressi che si affiancano alle lezioni. Durante il terzo anno di scuola ho seguito le attività formative presso il dipartimento di Dialisi Extracorporea dell' Ospedale San Michele di Cagliari, dove ho potuto appassionarmi alle tecniche dialitiche e approfondire tali argomenti.

\section{Percorso}

Nel 2013 ho avuto l'occasione di partecipare al 31st International Vicenza Course On Critical Care Nephrology. Il Congresso di Vicenza è un appuntamento annuale ormai consolidato, con la partecipazione di relatori, sia italiani sia internazionali, abili nell'esposizione delle loro presentazioni con grandi capacità comunicative, e che si avvalgono di spunti di riflessione sempre nuovi, riportando le ultime scoperte nei vari settori e le esperienze nei diversi paesi d'origine. Le presentazioni sono di elevato livello scientifico e orientate su argomenti innovativi, come ad esempio la pre- sentazione di nuove macchine di dialisi.

Per noi giovani, partecipare ai grandi congressi internazionali è sempre estremamente emozionante e culturalmente stimolante. Personalmente la mia partecipazione a questo congresso ha rappresentato un'importante opportunità per la mia formazione, perché mi ha consentito di programmare, e poi iniziare, un periodo di "fellowship" presso il dipartimento di Nefrologia e Dialisi dell'Ospedale San Bortolo di Vicenza. L'idea di questa fellowship è nata anche grazie alla precedente esperienza di un collega della mia stessa scuola di specializzazione; l'entusiasmo e l'ottima impressione trasmessi dai suoi racconti sono serviti da impulso. Proporre la mia candidatura per una fellowship a Vicenza è stato quindi un percorso relativamente facilitato dal punto di vista burocratico poiché la convenzione tra la scuola di specializzazione e l'ente ospitante era già impostata. In seguito, il primario della Nefrologia di Cagliari, il Prof. A. Pani, mi ha supportato in questo progetto.

Dopo l'accoglimento della mia domanda di frequenza, e dopo aver concluso l'opportuno iter burocratico per l'estensione dell'assicurazione, sono finalmente "approdata" a Vicenza nel Gennaio 2014, dove ho iniziato la mia avventura presso l'IRRIV (International Renal Research Institute of Vicenza). Tale istituto rappresenta il connubio tra esperienza clinica e ricerca scientifica: si tratta di un dipartimento annesso alla struttura ospedaliera che ospita i laboratori e gli spazi dedicati alla ricerca. La fre- 
quenza tradizionale presso il reparto o il dipartimento di Dialisi si affianca quindi al lavoro pomeridiano presso tale struttura che ospita ricercatori attratti in questo polo non Universitario dal fermento d'idee e dalla possibilità di portare avanti i propri progetti di ricerca. Questi lavori, in particolare, si articolano nelle diverse branche della nefrologia clinica, fisiopatologia renale, dialisi extracorporea, dialisi peritoneale, e trapianto renale, sovente intersecandosi in maniera complessa.

\section{Metodo vicenza}

La caratteristica fondamentale dell'IRRIV è il "metodo Vicenza" che si caratterizza per la collaborazione e la multidisciplinarietà. Precisamente, medici specialisti e specializzandi, farmacisti, statistici, ingegneri, sociologi, dietisti, biologi, e biotecnologi si affiancano condividendo le proprie competenze, esperienze, idee, e progetti. Questa combinazione di figure professionali permette di valutare ogni sfaccettatura dei vari progetti in corso, senza doversi limitare al solo aspetto puramente medico, ma esplorando a 360 gradi le problematiche e trovando soluzioni che altrimenti non sarebbero state possibili. Un fulgido esempio di ciò è stato la progettazione del "Carpe Diem", appositamente studiato di concerto tra medici e ingegneri per quella parte "orfana" della dialisi che è la sezione neonatale.

La valenza di tale istituto di ricerca è tale da attirare non solo medici specializzandi da diverse sedi Italiane (al momento della mia frequenza, oltre a me, erano presenti colleghi provenienti da Roma, Bari, Firenze, e Pisa) ma anche di diverse specialità, in particolare richiamando i colleghi delle scuole di Anestesia e Rianimazione che condividono lo studio sul danno renale acuto e sui trattamenti di sostituzione renale nel paziente critico. La collaborazione inoltre non si limita al solo territorio Italiano ma è internazionale; i "fellows" provengono da tutte le parti del mondo (i colleghi che mi hanno affiancato durante la fellowship provenivano da Messico, Cina, Brasile, India, Argentina, Germania, e Cile) e questa multiculturalità arricchisce sicuramente dal punto di vista scientifico e altresì dal punto di vista umano: confrontando culture, idee, religioni, e persino stili culinari (inaspettati e ottimi!) e, infine, mettendo alla prova la nostra conoscenza della lingua inglese, diventata ormai un "Esperanto" condiviso in tutto il mondo.

La frequenza presso questo centro mi ha permesso di approfondire la parte riguardante la "dialisi acuti". Per quanto breve sia la mia esperienza acquisita come specializzanda, la mia idea è che anche le piccole realtà ospedaliere devono potere intraprendere la dialisi in "acuto" e che i pazienti critici andrebbero trattati nel contesto di una terapia intensiva per poter essere monitorati costantemente e per poter ricevere le cure intensivistiche di cui necessitano. Sicuramente il modello della collaborazione tra nefrologo e intensivista è vincente, sia per la nostra professionalità sia, e soprattutto, per la qualità di trattamento che possiamo fornire al paziente. La partecipazione a multipli progetti di ricerca e la collaborazione con gli altri fellows ai loro progetti mi ha permesso di crescere e maturare in termini di capacità di "saper scri- vere un lavoro", ma soprattutto per come scrivere in maniera multidisciplinare e realizzare lavori tramite collaborazioni con figure professionali che, spesso, nelle realtà ospedaliere o universitarie, non si hanno a disposizione.

Non ultimo, il periodo a Vicenza mi ha permesso di partecipare all'organizzazione dell'edizione 2014 dell'International Vicenza Course: un evento di tale portata ha degli aspetti impegnativi, e la possibilità di intervenire in tale processo è stata decisamente istruttiva e appassionante.

Non cambierei nulla del mio percorso durante la Scuola di Specializzazione e della mia scelta di una fellowship in una struttura esterna alla sede di appartenenza: ritengo, infatti, sia fondamentale un periodo in una struttura e in una città differente, e non per forza all'estero! In Italia abbiamo molti reparti di eccellenza in cui poter ampliare il proprio bagaglio culturale di competenze specifiche e, contemporaneamente, prendere contatto con diverse realtà organizzative.

Esperienze di altri colleghi in altre sedi d'Italia confermano come la possibilità di compiere un'esperienza al di fuori del classico percorso formativo non sia sempre facile, e talvolta intoppi burocratici, come le convenzioni tra centri, possono precludere tali esperienze. Ritengo che, invece, queste possibilità debbano essere il più possibile agevolate e implementate per rendere la formazione la più ampia possibile.

\section{Conclusioni}

L'esperienza a Vicenza mi sta arricchendo in diverse direzioni; la ricerca in campo nefrologico mi ha sempre appassionato, non solo per il ruolo d'identificazione di nuove terapie per le patologie renali, ma anche il lato volto a implementare le conoscenze in tali ambiti. Questa esperienza nel Dipartimento di Ricerca non ha potuto che confermare la mia attitudine per tale campo, invogliandomi a proseguire il modello multidisciplinare qui incontrato anche nel mio futuro. Inoltre, anche l'aspetto della pratica clinica è decisamente stimolante, gli spunti formativi sono tanti e variegati.

\section{Riassunto}

La partecipazione a un congresso internazionale per uno specializzando è sempre emozionante e interessante. In particolare per me, la partecipazione al congresso di Vicenza ha rappresentato l'opportunità per progettare una frequenza al di fuori del normale percorso formativo, e accedere a un'esperienza di ricerca presso la Nefrologia/IRRIV dell' Ospedale San Bortolo di Vicenza. Ho potuto immergermi in una realtà multiculturale e interdisciplinare che mi ha affascinato ed entusiasmato per le sue applicazioni pratiche di ricerca e progettazione di macchine per dialisi.

Parole chiave: Insufficienza Renale Acuta, Ricerca Scientifica, Multidisciplinarietà

Ringraziamenti: Ringrazio tutti coloro che mi hanno permesso di vivere questa esperienza, in particolare il Prof. 
A. Pani, che ha creduto in questo mio progetto, e il Prof. C. Ronco, per avermi dato la possibilità di fare questa magnifica esperienza, non solo come scuola di Nefrologia ma anche come scuola di vita.

Dichiarazione di conflitto di interessi: L'Autore dichiara di non avere conflitto di interessi.

Contributi economici agli Autori: L'Autore dichiara di non avere ricevuto sponsorizzazioni economiche per la preparazione dell'articolo.
Indirizzo dell'Autore:

Dr.ssa Stefania Aresu

Dipartimento di Nefrologia

Dialisi e Trapianto di Rene

Ospedale San Michele

Via G. Peretti 2

09121 Cagliari

stefania.aresu@yahoo.it 